NACHRICHTEN

\section{New EC Website on ethics in research}

CORDIS, the European Commission's Research \& Development Information Service, is dedicating a specific section of its new Science and Society service to promote awareness of ethics in research and innovation activities.

The Fifth Framework programme supports strong ethical requirements for the financed research projects. The upcoming Framework Programme 2002 - 2006 will certainly reinforce this commitment for keeping progress in science in line with the ethical values of Member States and associated countries.

The site, part of the new "Science and Society" service, provides a central access to both European and international reference texts and legislative documents with regard to ethics in research. In addition, it offers direct links to the activities of the European Group on Ethics in Science and New Technologies currently led by Ms. Lenoir.

The service will be a valuable tool for esearchers and stakeholders to fully participate in and contribute to responsible research activities.

URL: http://www.cordis.lu/science-society

URL: http://www.cordis.lu/rtd2002/sciencesociety/ethics.htm

(Source: CORDIS focus)

》

\section{Hochschulgesellschaft Ober- hausen vergibt Stipendien für umweltwissenschaftliches Wei- terbildungsstudium}

Die Hochschulgesellschaft Oberhausen e.V. vergibt zum Wintersemester 2001/2002 und zum Sommersemester 2002 jeweils zwei Stipendien für das Interdisziplinäre Fernstudium Umweltwissenschaften (infernum). Bewerbun- gen sind ab sofort möglich. Das berufsbegle itende Fernstudium vermittelt Beschäftigten in Wirtschaft, Wissenschaft, Verwaltung und Verbänden Grundlagenwissen und Methoden aus Umweltwissenschaft und Umwelttechnik. Das innovative Weiterbildungsstudium wird gemeinsam von der FernUniversität Hagen und dem Fraunhofer-Institut für Umwelt-, Sicherheitsund Energietechnik UMSICHT in Oberhausen angeboten.

Die Stipendiat/inn/en werden nach fachlichen Kriterien ausgewählt. Darüber hinaus wird ein besonderes Engagement in Beruf oder Freizeit für die Belange des Umweltschutzes und einer nachhaltigen Entwicklungen erwartet. Im Rahmen der Stipendien trägt die Hochschulgesellschaft für eine maximale Förderdauer von vier Semestern jeweils die komple tten Studiengebühren in Höhe von etwa $1.250,00 €$ pro Semester.

Das viersemestrige Fernstudium infernum (www.fernuni-Hagen.de/umwelt) vermittelt Grundlagen- sowie Methodenwissen aus mehr als zehn umweltwissenschaftlichen Disziplinen. Hierbei zeichnet sich das Studium insbesondere durch die Vermittlung interdisziplinären Wissens aus. Rechts-, Wirtschafts- und Sozialwissenschaften sind ebenso vertreten wie umwelttechnische und naturwissenschaftliche Disziplinen. Die Themenpalette reicht von Abfallwirtschaft über betriebliches Umweltmanagement und Energieversorgung und Fragen des Klimawandels bis zur Wasserwirtschaft. Seit dem Start im November 2000 trifft das Studium bundesweit sowie im benachbarten Ausland bei Berufstätigen aus verschiedensten Professionen und Branchen auf großes Interesse.

(Pressemitteilung)

\section{Interessenten für ein Stipendium wenden sich bitten an}

Anja Gerstenmeier

Fraunhofer-Institut für Umwelt-, Sicherheits- und Energietechnik UMSICHT

Osterfelder Straße 3, 46047 Oberhausen

Tel.: 0208 / 8598 - 111

Fax: 0208 / 8598 - 290

E-Mail: ger@umsicht.fhg.de 